\title{
DISCUSSION ON HEIGHT SYSTEMS IN STEREOSCOPIC MAPPING USING THE ZY-3 SATELLITE IMAGES
}

\author{
Liping Zhao ${ }^{1, *}$, Xingke $\mathrm{Fu}^{1}{ }^{1}$, Guangbin Zhu ${ }^{1}$, Jianhua Zhang ${ }^{2}$, Chaobin Han $^{2}$, Lu Cheng ${ }^{2}$ \\ ${ }^{1}$ Satellite Surveying and Mapping Application Center, National Administration of Surveying, Mapping and Geoinformation, Beijing \\ 100048, China - (zhaolp, fxk, zhugb)@sasmac.cn \\ 2 The First Geodetic Surveying Brigade of National Administration of Surveying, Mapping and Geoinformation, Xi'an 710054, \\ China)-286214822@qq.com,417924975@qq.com,52539233@qq.com
}

Commission III, ICWG IV/III

KEY WORDS: ZY-3, Stereoscopic, Satellite, Mapping, Geoid, Ellipsoidal height, Orthometric height, Accuracy

\begin{abstract}
:
The ZY-3 is the civil high-resolution optical stereoscopic mapping satellite independently developed by China. It is mainly used for 1: 50,000 scale topographic mapping. One of the distinguishing features of the ZY-3 is that the panchromatic triplet camera can obtain thousands of kilometers of continuous strip stereo data. The working mode is suitable for wide-range stereoscopic mapping, in particular global DEM extraction. The ZY-3 constellation is operated in a sun-synchronous at an altitude 505 km, with a 10:30 AM equator crossing time and a 29-day revisiting period. The panchromatic triplet sensors have excellent base-to-height ratio, which is advantageous for obtaining good mapping accuracy.
\end{abstract}

In this paper the China quasi-geoid, EGM2008 and the height conversion method are discussed. It is pointed out that according to the current surveying and mapping specifications, almost all maps and charts use mean sea level for elevation. Experiments on bundle adjustment and DEM extraction with different height systems have been carried out in Liaoning Province of China. The results show that the similar accuracy can be obtained using different elevation system. According to the principle of geodesy and photogrammetry, it is recommended to use ellipsoidal height for satellite photogrammetric calculation and use the orthometric height in mapping production.

\section{INTRODUCTION}

The ZY-3 is the civil high-resolution optical stereoscopic mapping satellite independently developed by China. It is mainly used for 1: 50,000 scale topographic mapping. One of the distinguishing features of the ZY-3 is that the panchromatic triplet stereo camera can work continuously for long periods of time to obtain thousands of kilometers of continuous strip data. The working mode is suitable for wide-range stereoscopic mapping, in particular global DEM extraction.

The ZY-3 (02) satellite was successfully launched on May 30, 2016. The spacecraft is operated in a sun-synchronous at an altitude $505 \mathrm{~km}$, with a 10:30 AM equator crossing time and a 29-day revisiting period. The panchromatic triplet sensors, pointing forward, nadir, and backward with an angle of $22^{\circ}$, have excellent base-to-height ratio, which is advantageous for obtaining good horizontal and vertical accuracy. Table 1 and table 2 list the key specifications of the satellite and sensors.

The ZY-3 satellite has the advantages of high geolocation accuracy and structural stability. Based on the high precision gyros and star sensors on the satellite, the arc seconds level attitude measurement accuracy is obtained. The centimetres level orbit determination accuracy is achieved with a dualfrequency GPS receiver (Cao et al. 2012).
This paper introduces the coordinate frames and height systems of the ZY-3 (02) satellite image, ground control points and mapping specifications. The China quasi-geoid, EGM2008 and the height conversion method are discussed. It is pointed out that without ground control points, the vertical reference system used by RPC is WGS84 ellipsoid instead of the mean sea level (MSL). However, according to the current surveying and mapping specifications, almost all maps and charts use mean sea level for elevation. Therefore, the technical process of using "China refined quasi-geoid" or EGM2008 geoid to convert the ellipsoid height into the orthometric height is further explored.

\begin{tabular}{|c|c|}
\hline Orbital altitude & $505 \mathrm{~km}$ \\
\hline Orbital inclination & $97.421^{\circ}$ \\
\hline Revisit cycle (constellation) & 29 days \\
\hline Nodal Crossing & $10: 30 \mathrm{am}$ \\
\hline Off-Nadir Imaging & $<32^{\circ}$ \\
\hline
\end{tabular}

Table 1. The specifications of the satellite

\begin{tabular}{|c|c|c|c|}
\hline Sensors & $\begin{array}{c}\text { Nadir } \\
\text { Camera }\end{array}$ & $\begin{array}{c}\text { Forward } \\
\text { Camera }\end{array}$ & $\begin{array}{c}\text { Backward } \\
\text { Camera }\end{array}$ \\
\hline Spatial Resolution & $2.1 \mathrm{~m}$ & $2.5 \mathrm{~m}$ & $2.5 \mathrm{~m}$ \\
\hline Swath Width & $50 \mathrm{~km}$ & $52 \mathrm{~km}$ & $52 \mathrm{~km}$ \\
\hline Spectral Range & \multicolumn{3}{|c|}{ 450nm-800nm } \\
\hline Dynamic Range & \multicolumn{3}{|c}{ 10 bits per pixel } \\
\hline
\end{tabular}

Table 2. The specifications of the sensors

* Corresponding author 
Case studies using the ZY-3 (02) stereo satellite data for bundle adjustment and DEM extraction with different height systems have been carried out in Liaoning Province of China. The results show that there is no significant difference between horizontal and vertical accuracy of the schemes, no matter which coordinate frame and height system are used, which is less than 5 meters and meets the requirements of satellite design indexes.

\section{DATASETS}

Four ZY-3 stereo models in the same orbit with no cloud cover and clear features in Liaoning Province of China are used to analysis the precision. The images are obtained on the $27^{\text {th }}$ August 2016, and the GSD is around $2.5 \mathrm{~m}$. The base height ratio between backward and forward images is 0.88 , which is suitable for cartographical mapping and DEM extraction.

The experimental area covers about $200 \mathrm{~km}$ from north to south and about $50 \mathrm{~km}$ from east to west (Figure 1). Figure 2 is one of the thumbnails of the ZY-3(02) image data.

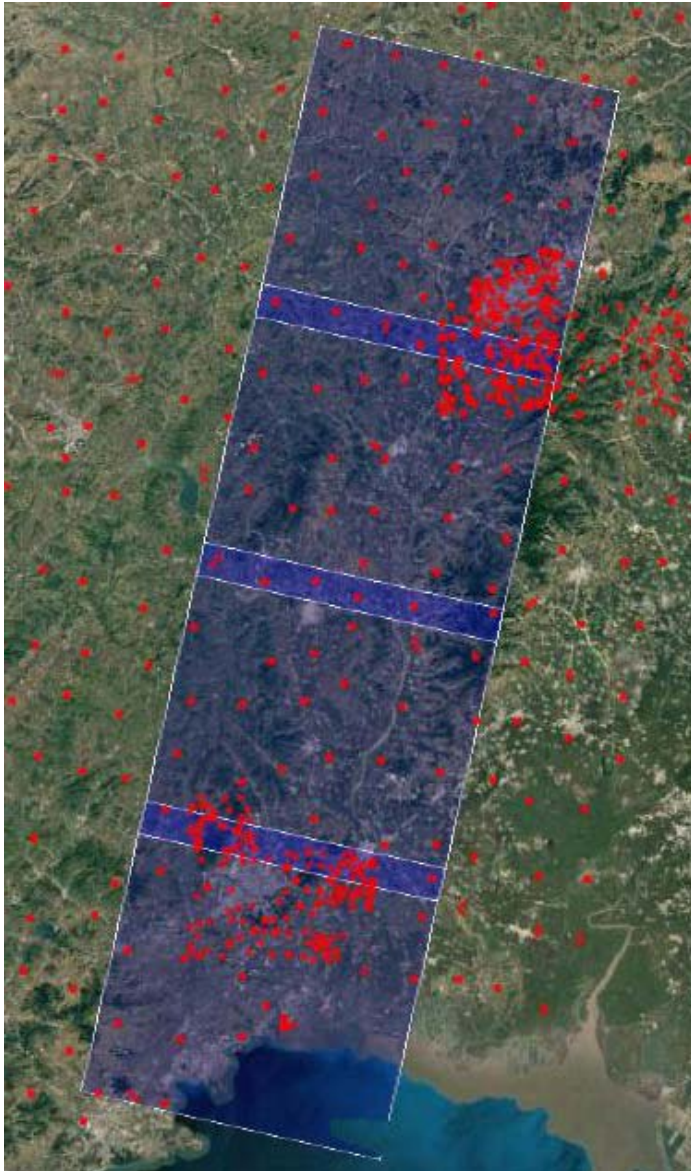

Figure 1. The schematic diagram of the experimental area and distribution of GCPs

In this experiment, 659 well-distributed ground control points over the four scenes as shown in Figure 1 ranging from $10 \mathrm{~m}$ to $432 \mathrm{~m}$ in height are used, and there are 133 control points in the bottom scene (Scene122). The ground coordinates of GCPs are measured in the field by RTK GPS based on the Continuously Operating Reference Stations (CROS) with centimeter-level accuracy in the horizontal and vertical coordinates. Their image coordinates are measured manually by stereoscopic models with accuracy reaching sub-pixel level. Most of the GCPs are grouped in sites of twin points, the accuracy and reliability are increased because of the redundancy of the groups of GCPs.

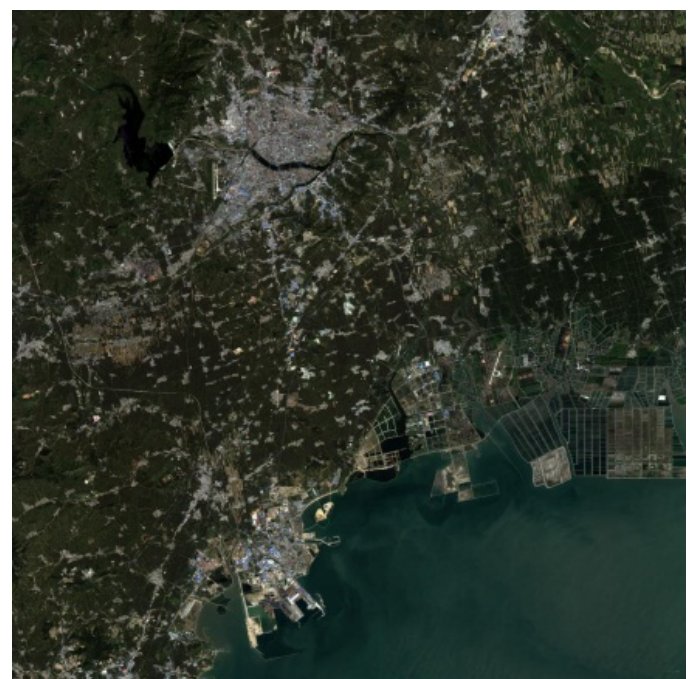

Figure 2. The thumbnail image of the ZY-3 in the experiment

\section{HEIGHT SYSTEMS}

Before analyzing the influence of different elevation systems on the accuracy of stereoscopic mapping, it is necessary to clearly explain and discuss the horizontal datum and vertical datum of the reference coordinate frame of the data used.

\subsection{Geoid and EGM}

The geoid is usually approximated by an "earth gravity model" (EGM) (EGM2008, 2018). The local refined China quasi-geoid and the EGM2008 published by the US National GeospatialIntelligence Agency (NGA) are used to convert heights between the orthometric height and ellipsoid height in the paper.

\subsubsection{China quasi-geoid}

In the experiment the local refined China quasi-geoid with centimetre-level accuracy in Liaoning Province of China is used to convert the ellipsoid height to the orthometric height above 1985 national elevation benchmarks of China.

Then the bundle adjustment and DEM extraction are realized from backward-forward image pairs of the ZY-3 satellite according to the Chinese 1:50000 scale national mapping specification.

\subsubsection{EGM2008}

The official Earth Gravitational Model EGM2008 (Figure 3) has been publicly released by the National GeospatialIntelligence Agency (NGA) EGM Development Team. This gravitational model is complete to spherical harmonic degree and order 2159, and contains additional coefficients extending to degree 2190 and order 2159.

Table 3 is a summary of the combined quantization and interpolation errors for the heights by the cubic interpolation, which is considerably accurate.

The accuracy of EGM2008 height anomalies achieves $20 \mathrm{~cm}$ in mainland, $12 \mathrm{~cm}$ in Central-East China, and even $9 \mathrm{~cm}$ in North China (Zhang et al. 2012). 


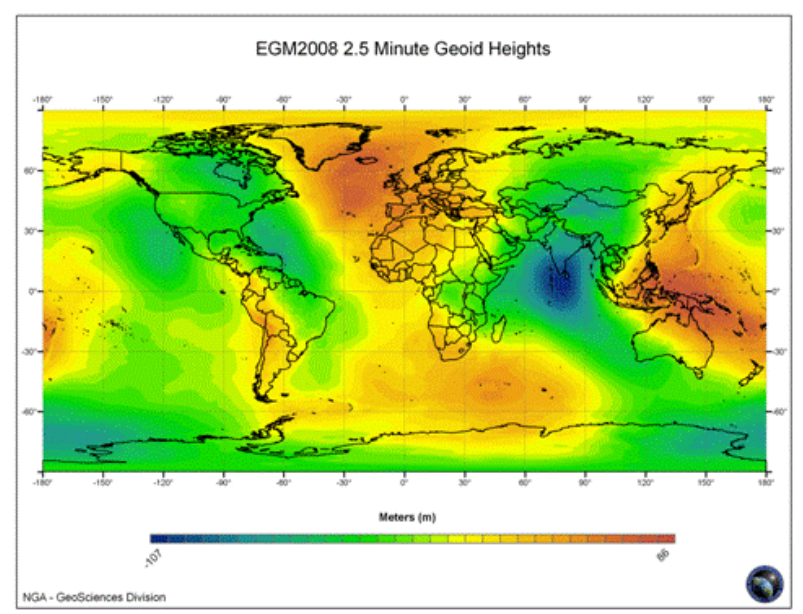

Figure 3. EGM2008 2.5 minute geoid heights

\begin{tabular}{|c|c|c|c|c|}
\hline name & geoid & grid & $\max$ & rms \\
\hline egm2008-5 & EGM2008 & $5^{\prime}$ & $0.294 \mathrm{~m}$ & $4.5 \mathrm{~mm}$ \\
\hline egm2008-2_5 & EGM2008 & $2.5^{\prime}$ & $0.031 \mathrm{~m}$ & $0.8 \mathrm{~mm}$ \\
\hline egm2008-1 & EGM2008 & $1^{\prime}$ & $0.0022 \mathrm{~m}$ & $0.7 \mathrm{~mm}$ \\
\hline
\end{tabular}

Table 3. Interpolation and quantization errors for geoid heights (https://geographiclib.sourceforge.io/)

\subsection{Height Conversion}

As shown in Figure 4, the geoid undulation above the ellipsoid, $\mathrm{N}$, can be used to convert a height above the ellipsoid, $\mathrm{h}$, to the corresponding orthometric height above the geoid (roughly the height above mean sea level), $\mathrm{H}$, using the relations as follows: (https://geographiclib.sourceforge.io/)

$$
\mathrm{h}=\mathrm{N}+\mathrm{H}
$$

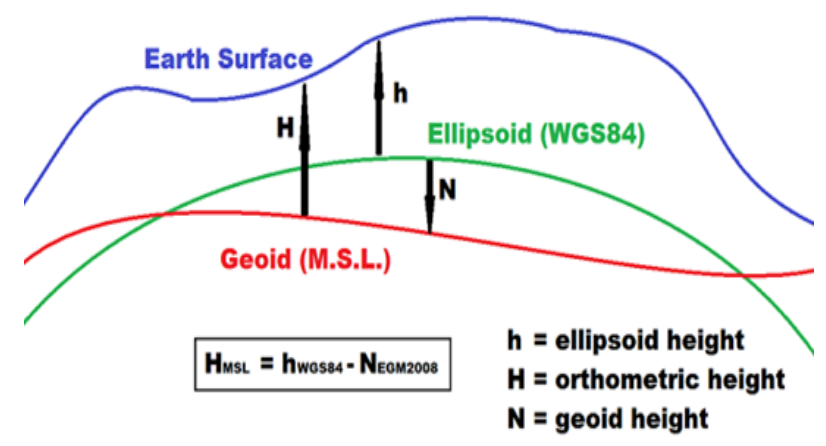

Figure 4. The schematic diagram of height conversion (https://www.eye4software.com/)

\subsection{RPCs provided with ZY-3}

Generally, For ZY-3 triplet image data, RPCs are generated by fitting an object space GCP grid. This grid is generated using the strict physical sensor model with the attitude and ephemeris data.

The orbit and ephemeris data of the ZY-3 satellite is determined by a dual-frequency GPS receiver (Cao et al. 2012). Therefore, the reference coordinate system of the ground in the RPC is based on the World Geodetic System 1984 used by the Global Positioning System and the elevation is the ellipsoid height above or below the WGS84 ellipsoid.

\subsection{Ground Control Data}

In the experiment the ground coordinates of GCPs are measured in the field by RTK GPS based on Continuously Operating Reference Stations (CROS).

Therefore, the horizontal and vertical coordinates are calculated in the framework of the CGCS2000, and the orthometric height above 1985 national elevation benchmarks of China is obtained using the local refined China quasi-geoid. At the same time, the object coordinates of the points are given in the Gauss Krüger projection in CGCS 2000 datum.

In order to study the accuracy of stereographic mapping under the WGS84 framework, EGM2008 is used to convert ellipsoidal heights and orthometric heights.

\section{EXPERIMENTS}

In this paper the vendor supplied RPCs and a sufficient number of well-distributed GCPs are used in bundle adjustment and DEM extraction, and affine compensation models in image space are used to improve the accuracy. The adjustment accuracy of GCPs with multiple elevation systems is compared and analyzed.

\subsection{Height Conversion}

According to the method described in Section 3, the heights of ground control points are converted to the orthometric heights above 1985 national elevation benchmarks of China (OH1985), the corresponding orthometric heights based on EGM2008 (OH2008) and the ellipsoid heights (EH1984) above the WGS84 ellipsoid respectively.

\subsection{Direct georeference}

Since the elevation in the RPCs is the ellipsoid height above the WGS84 ellipsoid, the GCPs with the WGS84 ellipsoid height is used to test the direct georeference accuracy.

For convenience, the four stereo models from north to south are named A, B, C, and D, respectively. The a-priori sensor accuracy of the ZY-3 triplet stereo images using rational function model by 659 independent check points is showed in Table 4.

From the analysis of the standard deviation data, it is found that the internal consistency of the ZY-3 data is very good, less than one GSD. However, in order to avoid the effects of the potential temporal drift of image locations and other minor affine deformations, an affine correction model in image space is used in the paper (Grodecki 2001, Jacobsen 2007).

\subsection{Bundle adjustment}

In this paper, the GCPs with the above-mentioned several elevation systems are respectively used to test the accuracy of the bundle adjustment.

First, in order to reliably assess the accuracy of the ZY-3 stereoscopic images and verify the accuracy of the control data, all 659 points with the WGS84 ellipsoid height are used as control points to calculate the bias and standard deviation (Table 5). 


\begin{tabular}{|c|c|c|c|c|}
\hline Models & Error & $\mathrm{N}(\mathrm{m})$ & $\mathrm{E}(\mathrm{m})$ & $\mathrm{H}(\mathrm{m})$ \\
\hline \multirow{2}{*}{$\begin{array}{c}\text { Model } \\
\mathrm{A}\end{array}$} & Bias & 19.248 & 12.323 & -7.498 \\
\cline { 2 - 5 } & Std.Dev. & 1.202 & 1.035 & 1.031 \\
\hline \multirow{2}{*}{$\begin{array}{c}\text { Model } \\
\mathrm{B}\end{array}$} & Bias & 19.147 & 12.638 & -8.936 \\
\cline { 2 - 5 } & Std.Dev. & 1.069 & 1.219 & 1.181 \\
\hline $\begin{array}{c}\text { Model } \\
\mathrm{C}\end{array}$ & Bias & 19.573 & 13.710 & -11.613 \\
\cline { 2 - 5 } & Std.Dev. & 1.049 & 1.133 & 1.238 \\
\hline $\begin{array}{c}\text { Model } \\
\text { D }\end{array}$ & Bias & 19.753 & 13.694 & -11.725 \\
\cline { 2 - 5 } & Std.Dev. & 1.204 & 1.145 & 1.409 \\
\hline
\end{tabular}

Table 4 . The a-priori sensor accuracy of the $\mathrm{ZY}-3$ triplet stereo images

\begin{tabular}{|c|c|c|c|c|}
\hline $\begin{array}{c}\text { Height } \\
\text { System }\end{array}$ & Error & $\mathrm{N}(\mathrm{m})$ & $\mathrm{E}(\mathrm{m})$ & $\mathrm{H}(\mathrm{m})$ \\
\hline \multirow{2}{*}{ EH1984 } & Bias & 0.000 & 0.000 & 0.000 \\
\cline { 2 - 5 } & Std.Dev. & 1.003 & 1.028 & 0.936 \\
\hline \multirow{2}{*}{ OH2008 } & Bias & 0.000 & 0.000 & 0.000 \\
\cline { 2 - 5 } & Std.Dev. & 1.003 & 1.027 & 0.929 \\
\hline \multirow{2}{*}{ OH1985 } & Bias & 0.000 & 0.000 & 0.000 \\
\cline { 2 - 5 } & Std.Dev. & 1.003 & 1.027 & 0.936 \\
\hline
\end{tabular}

Table 5. The bias and the standard deviation of all GCPs with the different height systems

Then, eight well-distributed points are used as ground control points, and the rest are used as independent checkpoints in model $\mathrm{D}$. The bundle block adjustment based on affine model is then performed. The bias error and standard deviation of GCPs/ICPs after the adjustment are shown in Table 6 .

\begin{tabular}{|c|c|c|c|c|c|c|c|}
\hline \multirow{2}{*}{$\begin{array}{l}\text { Height } \\
\text { System }\end{array}$} & \multirow{2}{*}{ Error } & \multicolumn{2}{|c|}{$\mathrm{N}(\mathrm{m})$} & \multicolumn{2}{c|}{$\mathrm{E}(\mathrm{m})$} & \multicolumn{2}{c|}{$\mathrm{H}(\mathrm{m})$} \\
\cline { 3 - 8 } & & GCP & ICP & GCP & ICP & GCP & ICP \\
\hline \multirow{2}{*}{ EH1984 } & Bias & 0.00 & -0.16 & 0.00 & -0.20 & 0.00 & 0.11 \\
\cline { 2 - 8 } & Std.Dev. & 0.59 & 1.21 & 1.04 & 1.14 & 0.89 & 1.27 \\
\hline \multirow{2}{*}{ OH2008 } & Bias & 0.00 & -0.16 & 0.00 & -0.20 & 0.00 & 0.11 \\
\cline { 2 - 8 } & Std.Dev. & 0.59 & 1.21 & 1.04 & 1.14 & 0.92 & 1.27 \\
\hline \multirow{2}{*}{ OH1985 } & Bias & 0.00 & -0.16 & 0.00 & -0.20 & 0.00 & 0.11 \\
\cline { 2 - 8 } & Std.Dev. & 0.59 & 1.21 & 1.04 & 1.14 & 0.90 & 1.27 \\
\hline
\end{tabular}

Table 6 . The bias and the standard deviation of 8 GCPs and 125 ICPs with the different height systems

It is found that there is almost no difference in adjustment accuracy using different elevation systems, and the planimetric and altimetric accuracy of all cases is better than one GSD.

\subsection{Mapping production}

The stereo model D is further used to extract the DEM, make the digital ortho-image map (DOM), and draw the digital topographic map. The DEM, DOM and topographic map are produced according to the national mapping specification.

Because the adjustment accuracy of using different elevation systems is basically the same, this article only carried out the DEM generation based 1985 national elevation benchmarks of China according to the Chinese 1:50000 scale DEM national specification and the space grid is $10 \mathrm{~m}$ for Digital Surface Model (DSM) and 25m for Digital Terrain Model (DTM).
In order to eliminate serious errors in some difficult areas (such as reservoirs, lakes, rivers, etc.), automatic filtration and human-computer interaction stereo editing are applied in the DEM production process.

The DSM and DTM extracted from backward-forward image pairs of the ZY-3 satellite are shown in Figure 5. Table 7 lists the RMSE and maximum error of the check points, which meet the national mapping requirements of 1:50,000 scale. Zhao et al 2018 gives a detailed discussion and analysis of the accuracy of DSM and DTM.
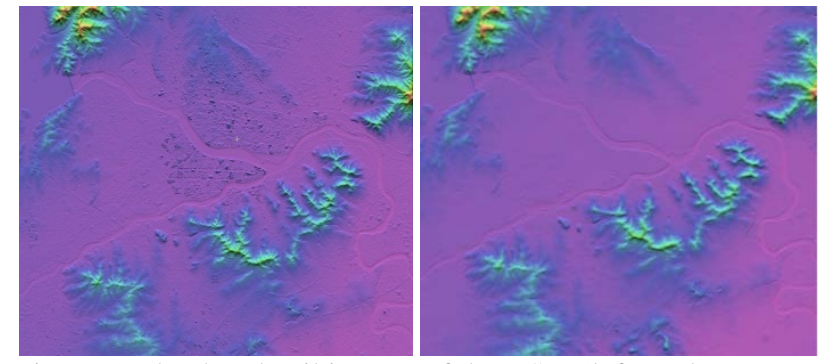

Figure 5. The thumbnail images of the DSM (left) and DTM (right)

\begin{tabular}{|c|c|c|c|c|c|c|}
\hline Product & CPs & Grid & Bias & Std.Dev. & RMSE & Max err. \\
\hline DSM & 24 & 10 & 0.203 & 1.473 & 1.487 & 2.796 \\
\hline DTM & 24 & 25 & -0.967 & 1.401 & 1.702 & 3.409 \\
\hline
\end{tabular}

Table 7. The DSM and DTM accuracy (unit: meter)

In addition to the DSM and DTM, digital topographic map (Figure 6) and ortho-image map (Figure 7) are also produced, and their accuracy meets national mapping requirements.

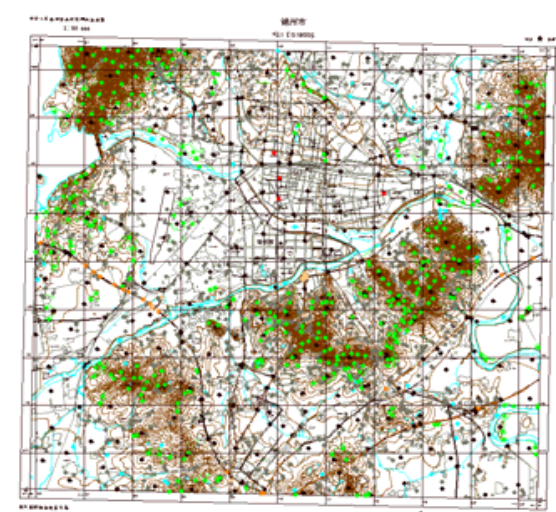

Figure 6. The thumbnail image of the digital topographic map

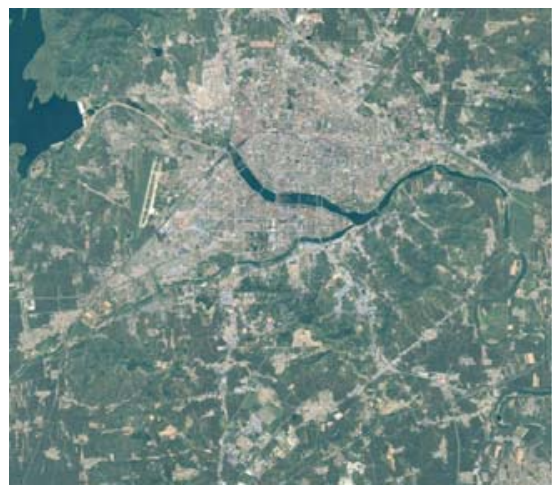

Figure 7. The thumbnail ortho-image 


\section{DISCUSSION}

The geoid surface is irregular, but considerably smoother than Earth's physical surface. According to Gauss, who first described it, it is the "mathematical figure of Earth", a smooth but highly irregular surface whose shape results from the uneven distribution of mass within and on the surface of Earth (Wiki, 2018).

A reference ellipsoid is a mathematically defined surface that approximates the geoid. Because of their relative simplicity, reference ellipsoids are used as a preferred surface on which geodetic network computations are performed and point coordinates such as latitude, longitude, and elevation are defined (Wiki, 2018).

Photogrammetry is the science of making measurements from photographs; it uses methods from many disciplines, including optics and projective geometry. (Wiki, 2018)

Therefore, the reference ellipsoid and photogrammetry are geometric quantities relative to the geoid representing the physical quantity.

Although in the experiments in this paper and most of the applications, the similar accuracy can be obtained using different elevation system. However, based on the principle of geodesy and photogrammetry, it is recommended to use ellipsoidal height for satellite photogrammetric processing to calculate the image and ground coordinates. In the production of mapping products, projection transformation and elevation conversion are performed according to the mapping specification.

\section{CONCLUSION}

In the paper the China quasi-geoid, EGM2008 and the height conversion are discussed. The bundle adjustment and DEM extraction based different height systems using the ZY-3 (02) stereo satellite data have been carried out. The results show that there is no significant accuracy difference with different height systems.

However, according to the principle of geodesy and photogrammetry, this paper proposes to use the WGS84 ellipsoid height in the calculation of satellite stereoscopic measurements and use the orthometric height in mapping production.

\section{REFERENCES}

Cao Haiyi, Liu Xigang, Li Shaohui and Zhang Xinwei, 2012. ZY-3 Satellite Remote Sensing Technology, Spacecraft Recovery \& Remote Sensing, 33, pp. 7-16

EGM2008, 2018. http://earth-info.nga.mil/ (10 June 2018)

Grodecki, J., 2001. IKONOS Stereo Feature Extraction - RPC Approach, ASPRS annual conference St. Louis 2001

Jacobsen, K., 2007. Orientation of high resolution optical space images, ASPRS annual conference, Tampa 2007

Wiki, 2018. https://en.wikipedia.org/wiki/ (10 June 2018)
Zhang Chuanyin, Guo Chunxi, Chen Junyong, Zhang Liming and Wang Bin, 2009. EGM 2008 and its application analysis in Chinese mainland, Acta Geodaetica et Cartographica Sinica, 38, pp. 283-289

Zhao Liping, Fu Xingke, Dou Xianhui, Liu hui and Fang Zhi, 2018. Comparison and analysis of accuracy of elevation extraction based on the ZY-3 01 and 02 satellites stereoscopic images, ISPRS TC III Symposium 2018, Beijing, China. (Accepted) 